\title{
Perfil clínico-epidemiológico de pacientes com insuficiência cardíaca internados em hospital público de São Luís-MA
}

\author{
Clinical and epidemiological profile of patients with heart failure admitted to a public \\ hospital of São Luís-MA
}

Costa, Louise Christine de Castro ${ }^{1}$; Ribeiro, Maria Jacqueline Silva ${ }^{2}$; Costa, Vera Lívia Xavier de Castro ${ }^{3}$; Lima, Rodrigo Trabulsi ${ }^{4}$

Resumo: Objetivos: Comparar o perfil clínico-demográfico de portadores de IC internados em um hospital público de São Luís no ano de 2017. Identificar os fatores de risco para doenças cardiovasculares. Determinar a prevalência de IC com fração de ejeção preservada (ICFEP) e de IC com fração de ejeção reduzida (ICFER). Determinar a frequência de pacientes com tratamento adequado de acordo com as diretrizes atuais. Material e Métodos: Foram analisadas as seguintes variáveis: idade, sexo, dias de internação hospitalar, sinais e sintomas dos critérios de Frammingham, fatores de risco/comorbidades (Diabetes, hipertensão, etilismo, tabagismo, anemia, doença renal crônica e outros), exames (hemograma, $\mathrm{Na}+$, ureia, creatinina, e e fração de ejeção - FE) e medicamentos prescritos na internação hospitalar. Resultados e Discussão: Neste estudo, verificouse que a idade média foi similar ao estudo BREATH (64 \pm 16 anos). O período de internação hospitalar foi extenso nesta casuística. Estudos como ADHERE tem descrito que os DIH de pacientes com IC são em média de 4 a 5 dias para os casos não graves e de 9 a 11 dias para aqueles mais graves. Quanto a Fração de Ejeção (FE), observou-se maior prevalência de FE preservada nas mulheres em relação aos homens, que tiveram maiores taxas de FE reduzida. Correlacionando-se as variáveis clínicodemográficas com os dias de internação hospitalar (DIH), verificou-se associação estatisticamente significativa para hiponatremia e FE reduzida, e menor correlação com sexo, idade e anemia. Conclusão: Conclui-se que a IC com fração de ejeção reduzida (ICFER) foi mais associada ao sexo masculino, internação prolongada, idade avançada e sinais e sintomas dos critérios de Frammingham. Quanto ao tratamento empregado na ICFER, predominou o uso de IECA ou BRA, espironolactona e betabloqueador.

Palavras chaves: Insuficiência Cardíaca. Fração de Ejeção. Fatores de Risco. Tratamento.

Abstract: Objectives: To compare the clinical and demographic profile of patients with HF admitted to a public hospital in São Luís in 2017. To identify risk factors for cardiovascular diseases. To determine the prevalence of HF with preserved ejection fraction (HFPS) and HF with reduced ejection fraction (HFFR). Determine the frequency of patients receiving appropriate treatment according to current guidelines. Materials and Methods: The following variables were analyzed: age, sex, hospitalization days, signs and symptoms of the Frammingham criteria, risk factors / comorbidities (Diabetes, hypertension, alcoholism, smoking, anemia, chronic kidney disease and others), exams (hemogram, $\mathrm{Na}$ + , urea, creatinine, and ejection fraction - FE) and medications prescribed at hospital admission. Results and Discussion: In this study, it was found that the mean age was similar to the BREATH study (64 \pm 16 years). The length of hospital stay was extensive in this series. Studies such as ADHERE have described that the IHDs of patients with HF are on average 4 to 5 days for non-severe cases and 9 to 11 days for those more severe. Regarding ejection fraction (EF), there was a higher prevalence of preserved EF in women than in men, which had higher EF rates. Correlating the clinical-demographic variables with the days of hospitalization (IHD), there was a statistically significant association for hyponatremia and reduced EF, and a lower correlation with sex, age and anemia. Conclusion: It was concluded that HF with reduced ejection fraction (HFUT) was more associated with males, prolonged hospitalization, advanced age and signs and symptoms of the Frammingham criteria. Regarding the treatment used in HFUT, the use of ACE inhibitors or BRA, spironolactone and beta-blocker predominated.

Key Words: Heart Failure. Fraction of Ejection. Risk factors. Treatment.

\footnotetext{
${ }^{1}$ Médica formada Universidade Ceuma. São Luís, Maranhão, Brasil.

2 Médica Cardiologista, docente da Universidade Ceuma.São Luís, Maranhão, Brasil.

${ }^{3}$ Médica Cardiologista (UFMA). São Luís, Maranhão, Brasil.

${ }^{4}$ Médico (UNICEUMA). São Luís, Maranhão, Brasil.

Endereço do autor correspondente: Louise Christine de Castro Costa E-mail: louisecosta_@hotmail.com. Endereço: Avenida dos Holandeses Quadra: 11 Casa:14 Parque Atlântico Bairro: Olho d'água, São Luís-MA, CEP: 65065-180 Fone: (98) 98115-6181.
} 


\section{Introdução}

As doenças cardiovasculares (DCV) constituem a principal causa de morte no Brasil ${ }^{1}$ e no mundo ${ }^{2}$. A insuficiência cardíaca (IC) participa de maneira expressiva por ser a via final comum da maioria das doenças que acometem o coração ${ }^{3}$, sendo uma síndrome clínica complexa de caráter sistêmico, definida como disfunção cardíaca que ocasiona inadequado fornecimento sanguíneo para suprir as necessidades metabólicas teciduais ou que só consegue fazê-lo com elevadas pressões de enchimento ${ }^{4}$.

A IC é uma doença com características de malignidade, com mortalidade superior à do câncer de mama nas mulheres e a do câncer de próstata nos homens ${ }^{5}$.

A melhora no cuidado de doenças como a hipertensão arterial sistêmica (HAS), diabetes mellitus (DM), dislipidemias e de outros fatores de risco para IC, bem como os avanços tecnológicos no tratamento da síndrome coronariana aguda (SCA) exercem grande influência na prevalência da IC. Isso tem permitido que as pessoas vivam mais tempo com os fatores de risco e possam desenvolver a doença posteriormente ${ }^{6}$.

Deve-se considerar ainda a incidência da IC secundária ao tratamento quimioterápico e radioterápico de muitas neoplasias ${ }^{7}$.

A disponibilidade de exames complementares de imagem não invasivos e a maior conscientização médica sobre a doença proporcionam diagnósticos mais frequentes $e$ precisos, e constituem outros fatores a serem considerados na epidemiologia da $\mathrm{IC}^{8}$

Segundo dados atuais da American Heart Association (AHA) estima-se a prevalência de IC nos Estados Unidos em 5,1 milhões de indivíduos (2007-2012). As perspectivas demonstram que a prevalência dessa doença aumentará $46 \%$ de 2012-2030, resultando em mais de 8 milhões de pessoas acima de 18 anos de idade com IC .

A IC é tão prevalente em homens como em mulheres, e essa prevalência aumenta com a idade em ambos os sexos. Os homens têm uma maior prevalência de IC até 75 anos de idade. Acima dessa faixa etária, passa a haver predomínio das mulheres. Portanto, o envelhecimento crescente da população tem papel importante na epidemiologia da doença ${ }^{10}$.

No Brasil, dados do DATASUS de 2012 demonstram que a IC é a terceira causa de hospitalização em todas as idades, e constitui a principal causa nos pacientes com idade superior a 60 $\operatorname{anos}^{11}$.

A recorrência de readmissões é particularmente elevada após uma primeira hospitalização por IC. Entre pacientes norte americanos com mais de 70 anos, por exemplo, aproximadamente $60 \%$ são readmitidos em 90 dias $^{12}$. A mortalidade da IC aumenta com a gravidade clínica, podendo atingir até $80 \%$ em dois anos para pacientes com classe funcional IV pela New York Heart Association (NYHA).

Dessa maneira, o aumento da prevalência com o avançar da idade, associado à recorrência de internações hospitalares e, por outro lado, o fato de cada vez mais adultos em idade produtiva serem acometidos, faz da IC um grande ônus à sociedade. Ainda em relação à qualidade de vida dos pacientes, deve-se considerar 0 aspecto extremamente limitante dessa doença. Assim 0 impacto socioeconômico desta doença, que já é grande, se tornará ainda maior ${ }^{13}$.

Apesar da frequência e da gravidade da doença, os recursos aplicados nas pesquisas de novas terapias ainda são baixos em relação 
às pesquisas de outras áreas, como a do câncer ${ }^{14}$.

No Brasil, são escassos os estudos que avaliam de forma compreensiva e prospectiva as características demográficas, clínicas e prognósticas de pacientes que são admitidos com diagnóstico clínico de $\mathrm{IC}^{15}$.

Considerando a necessidade de mais estudos em populações específicas para obter o diagnóstico e o tratamento corretos do doente com IC, foi despertado o interesse em realizar esse estudo cujo objetivo foi identificar - perfil clínico-epidemiológico e terapêutico de pacientes com IC internados em hospital público de São Luís - MA no ano de 2017.

\section{Material e métodos}

Trata-se de um estudo retrospectivo, realizado no Hospital De Referência Estadual de Alta Complexidade Dr. Carlos Macieira, em São Luís do Maranhão (MA) no ano de 2017.

A coleta de dados foi realizada por meio da análise de prontuários de pacientes internados com diagnóstico de IC, no período de janeiro a outubro de 2017. Foram analisadas as seguintes variáveis: idade, sexo, cor da pele, dias de internação hospitalar, fatores de risco (HAS, DM, tabagismo e etilismo), sinais e sintomas dos critérios de Framingham, comorbidades como: Doença Renal Crônica (DRC), Doença Arterial Coronariana (DAC) e anemia, pressão arterial sistólica (PAS) e pressão arterial diastólica (PAD) de admissão, sódio e potássio séricos, ureia, creatinina, fração de ejeção do ventrículo esquerdo (FE) e medicamentos prescritos na internação hospitalar.

Foram incluídos prontuários de pacientes com diagnóstico de IC pelos critérios

diagnósticos

de

Framingham $^{16}$, de ambos os sexos e com idade igual ou superior a 18 anos internados na enfermaria de cardiologia.

Foram excluídos prontuários de pacientes que não preencheram os critérios diagnósticos de Framingham para IC, prontuários incompletos e prontuários de pacientes grávidas.

Os pacientes com IC foram separados em dois grupos para fins de análise, de acordo com o tipo de disfunção determinado pela fração de ejeção do ventrículo esquerdo:1) Insuficiência cardíaca com fração de ejeção reduzida (ICFER) - FE < 50\%; e 2) Insuficiência cardíaca com fração de ejeção normal (ICFEN) - FE $\geq 50 \%{ }^{17}$.

$\mathrm{Em}$ relação à terapêutica empregada no tratamento para a IC, foi avaliada a administração de inibidores da enzima conversora da angiotensina (IECA), bloqueadores dos receptores da angiotensina (BRA), espironolactona e betabloqueadores, que são medicações que comprovadamente reduzem a mortalidade da ICFER.

Todos os dados desta pesquisa foram organizados em gráficos e tabelas descritivas para melhor visualização dos mesmos. Para o cálculo estatístico, foi utilizado o software SPSS v. 19, admitindo-se um nível de significância de 0,05. Características demográficas e clínicas foram comparadas entre os grupos de pacientes de Insuficiência cardíaca (IC) com Fração de Ejeção (FE) Reduzida e Preservada, sendo as variáveis qualitativas avaliadas pelos testes do Qui-quadrado e Qui-quadrado com correção de Yates, e as quantitativas pela ANOVA one-way. Para atendimento aos pressupostos da ANOVA foram analisadas previamente a normalidade dos dados (Teste de Shapiro-Wilk) e a homogeneidade de variâncias (Teste de Levene). A 
prevalência dos critérios de Framingham dentro dos grupos de FE foram verificadas também pelos testes do Qui-quadrado e Qui-quadrado com correção de Yates. Possíveis relações entre os Dias de Internação Hospitalar (DIH) com as variáveis qualitativas (sexo, faixa etária, anemia, FE e hiponatremia) foram conferidas pela Correlação Linear de Spearman. Também caracterizou-se quantitativamente as medicações empregadas em pacientes com $\mathrm{FE}$ reduzida.

A pesquisa foi aprovada pelo Comitê de Ética em Pesquisa (CEP) do Hospital São Domingos (HSD), com número de parecer 2.169.145.

\section{Resultados}

No período do estudo foram analisados 61 prontuários de portadores de IC internados no Hospital Dr. Carlos Macieira. Destes, 57.5\% apresentavam ICFER e $44.3 \%$ ICFEP. A tabela 1 mostra as características demográficas, clínicas e o tempo de internação da amostra estudada de acordo com o tipo de IC.

Em relação aos fatores prognósticos e determinantes para 0 período de internação hospitalar, não se observou correlação entre as variáveis estudadas e o tempo de internação hospitalar (Tabela 2).

Quanto aos critérios de Framingham para IC, verificou-se que a maioria dos sinais e sintomas foram mais prevalentes na ICFER que na ICFEP (Tabela 3)

Em relação ao tratamento na ICFER, verificou-se que $35.3 \%$ dos pacientes faziam uso de inibidores da enzima conversora de angiotensina (IECA) ou bloqueadores do receptor da angiotensina (BRA) + betabloqueador + espironolactona (Tabela 4).

\section{Discussão}

$\mathrm{Na}$ amostra estudada a frequência de ambos os sexos foi homogênea, semelhante ao encontrado no Brazilian Registry of Heart Failure (BREATH) ${ }^{18}$, que incluiu $60 \%$ de mulheres e $40 \%$ de homens, contrastando com a grande maioria dos estudos sobre IC como: Studies Of Left Ventricular Dysfunction (SOLVD) ${ }^{19}$, The Cardiac Insufficiency Bisoprolol Study $(\mathrm{CIBIS})^{20}$ e Digitalis Investigation Group (DIG) ${ }^{21}$ que incluíram poucas mulheres. A IC é tão prevalente em homens como em mulheres, e essa prevalência aumenta com a idade em ambos os sexos. Os homens têm uma maior prevalência de IC até 75 anos de idade, mas acima dessa faixa etária passa a haver predomínio de mulheres, ressaltando a necessidade de inclusão de mulheres nos estudos sobre IC. ${ }^{17}$

Neste estudo, verificou-se que a idade média foi similar ao estudo BREATH $\left(64 \pm 16\right.$ anos) ${ }^{18}$. O registro BREATH é o primeiro a incluir uma ampla amostra de pacientes hospitalizados com IC descompensada de diferentes regiões do Brasil.

De acordo com Barreto et al, dados epidemiológicos qualificam a idade > 65 anos como fator predisponente para o aparecimento de IC. ${ }^{22}$ Dois grandes registros de IC, Acute Descompensated Heart Failure National Registry (ADHERE) ${ }^{23}$ e Outcomes of a Prospective Trial of Intravenous Milrinone for Exacerbations of Chronic Heart Failure (OPTMIZE$\mathrm{HF})^{24}$ mostraram a média de idade dos pacientes de 72-73 anos. Um estudo publicado recentemente encontrou alta taxa anual de internação por IC em homens, principalmente naqueles com idade superior a 65 anos $^{25}$. A amostra estudada apresentou idade média inferior à dos estudos citados, o que pode ser atribuído às dificuldades de 
Tabela 1: Características demográficas e clínicas em função do tipo de Insuficiência Cardíaca.

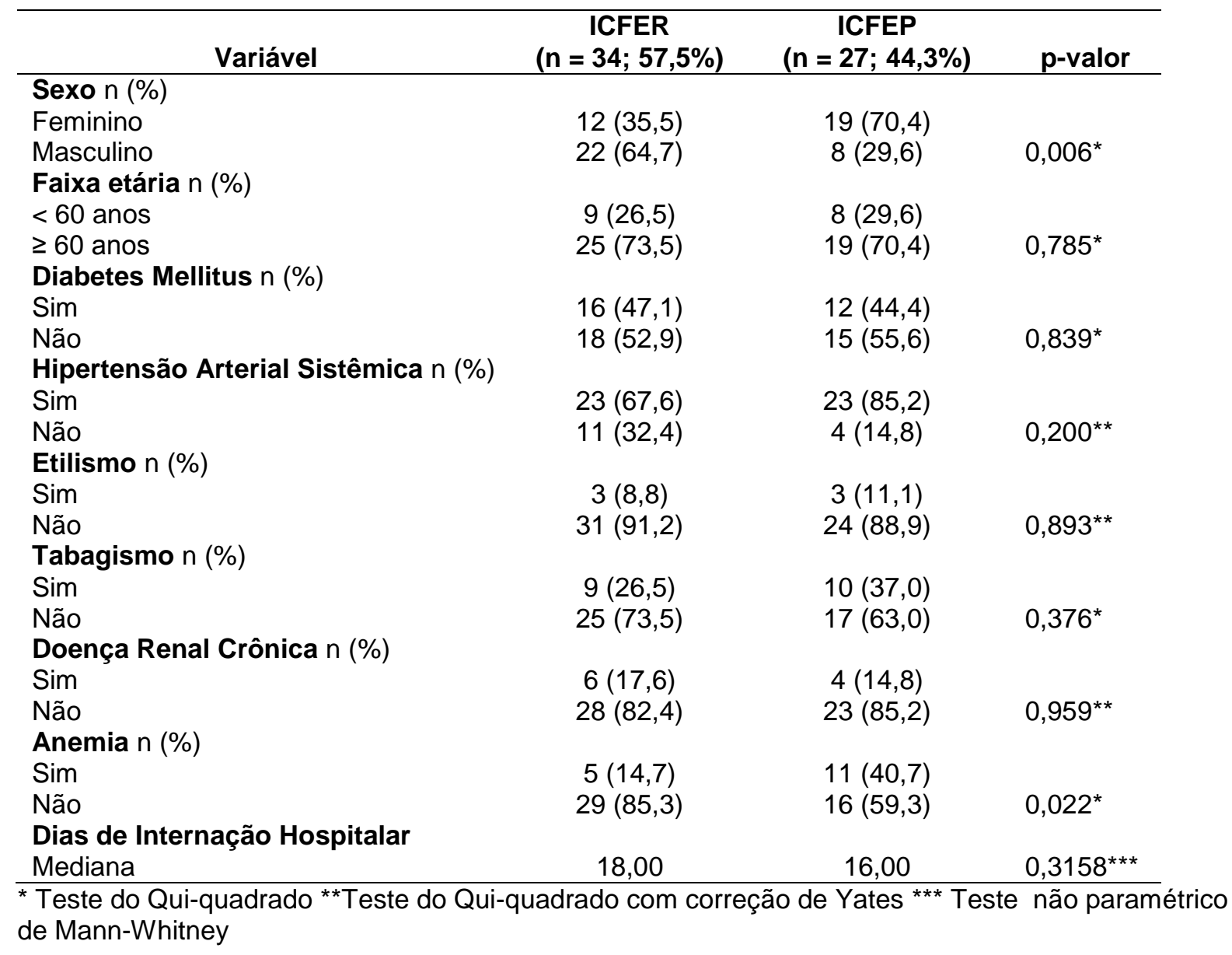

Tabela 2: Correlações entre os Dias de Internação Hospitalar (DIH) e as características demográficas e clínicas (anemia, fração de ejeção e hiponatremia).

\begin{tabular}{lcc}
\hline \multicolumn{1}{c}{ Variável } & $\begin{array}{c}\text { DIH } \\
\text { (Coeficiente) }\end{array}$ & p-valor \\
\hline Sexo & 0,036 & $0,781^{*}$ \\
Faixa etária & $-0,288$ & $0,024^{*}$ \\
Anemia & 0,070 & $0,592^{*}$ \\
Hiponatremia & 0,023 & $0,858^{*}$ \\
Fração de ejeção & $-0,101$ & $0,437^{*}$ \\
\hline \multicolumn{2}{c}{${ }^{*}$ Correlação Linear de Spearman } \\
\end{tabular}


Tabela 3:Prevalência dos critérios de Framingnham em função do tipo de Insuficiência Cardíaca.

\begin{tabular}{|c|c|c|c|}
\hline Critérios de Framingham & $\begin{array}{c}\text { ICFER } \\
(\mathrm{n}=34 ; 57,5 \%)\end{array}$ & $\begin{array}{c}\text { ICFEP } \\
(n=27 ; 44,3 \%)\end{array}$ & p-valor \\
\hline \multicolumn{4}{|l|}{$\begin{array}{l}\text { Dispneia paroxística noturna } \\
\mathrm{n}(\%)\end{array}$} \\
\hline Sim & $21(61,8)$ & $18(66,7)$ & \\
\hline Não & $13(38,2)$ & $9(33,3)$ & $0,962^{*}$ \\
\hline \multicolumn{4}{|l|}{ Estase jugular $\mathrm{n}(\%)$} \\
\hline Sim & $7(20,6)$ & $2(7,4)$ & \\
\hline Não & $27(79,4)$ & $25(92,6)$ & $0,281^{* *}$ \\
\hline \multicolumn{4}{|l|}{ Cardiomegalia $\mathrm{n}(\%)$} \\
\hline Sim & $11(32,4)$ & $6(22,2)$ & \\
\hline Não & $23(67,6)$ & $21(77,8)$ & $0,381^{*}$ \\
\hline \multicolumn{4}{|l|}{ Estertores pulmonares n (\%) } \\
\hline Sim & $20(58,8)$ & $13(48,1)$ & \\
\hline Não & $14(41,2)$ & $14(51,9)$ & $0,406^{*}$ \\
\hline \multicolumn{4}{|l|}{ Edema agudo de pulmão $n(\%)$} \\
\hline $\operatorname{Sim}$ & $7(20,6)$ & $2(7,4)$ & \\
\hline Não & $27(79,4)$ & $25(92,6)$ & $0,281^{*}$ \\
\hline \multicolumn{4}{|l|}{ Edema BI MMII n (\%) } \\
\hline $\operatorname{Sim}$ & $7(20,6)$ & $21(77,8)$ & \\
\hline Não & $27(79,4)$ & $6(22,2)$ & $0,827^{\star}$ \\
\hline \multicolumn{4}{|l|}{ Tosse noturna $\mathrm{n}(\%)$} \\
\hline Sim & $14(41,2)$ & $8(29,6)$ & \\
\hline Não & $20(58,8)$ & $19(70,4)$ & $0,351^{*}$ \\
\hline \multicolumn{4}{|l|}{$\begin{array}{l}\text { Dispneia para esforço habitual } n \\
(\%)\end{array}$} \\
\hline Sim & $32(94,1)$ & $25(92,6)$ & \\
\hline Não & $2(5,9)$ & $2(7,4)$ & $0,778^{\star \star}$ \\
\hline \multicolumn{4}{|l|}{ Derrame pleural n (\%) } \\
\hline Sim & $7(20,6)$ & $7(25,9)$ & \\
\hline Não & $27(79,4)$ & $20(74,1)$ & $0,622^{*}$ \\
\hline \multicolumn{4}{|l|}{ Taquicardia n (\%) } \\
\hline Sim & $6(17,6)$ & $5(18,5)$ & \\
\hline Não & $28(82,4)$ & $22(81,5)$ & $0,930^{*}$ \\
\hline
\end{tabular}

Tabela 4: Tipos terapêuticos utilizados em pacientes com Insuficiência Cardíaca com Fração de Ejeção Reduzida (ICFER).

\begin{tabular}{lc}
\multicolumn{1}{c}{ Terapêutica } & $\mathbf{N}(\%)$ \\
\hline BRA ou IECA + BETABLOQUEADOR +ESPIRONOLACTONA & $12(35,3)$ \\
BRA ou IECA + ESPIRONOLACTONA & $16(47,1)$ \\
BRA Ou IECA + BETABLOQUEADOR & $15(44,1)$ \\
ESPIRONOLACTONA + BETABLOQUEADOR & $17(50,0)$ \\
BRA Ou IECA & $22(64,7)$ \\
BETABLOQUEADOR & $25(73,5)$ \\
ESPIRONOLACTONA & $22(64,7)$ \\
\hline
\end{tabular}


acesso ao tratamento dos fatores etiológicos na região, ocasionando rápida progressão da doença.

O período de internação hospitalar foi extenso nesta casuística. Estudos como ADHERE ${ }^{(23)}$ e 0 EuroHeart Failure Survey II (EHFS II) ${ }^{26}$ têm descrito que os pacientes com IC ficam internados em média de 4 a 5 dias para os casos não graves e de 9 a 11 dias para aqueles mais graves. Um estudo que comparou padrão de desfecho de pacientes com IC no Brasil e nos Estados Unidos verificou que os pacientes norte-americanos com IC tiveram uma permanência hospitalar média de 5 dias, enquanto os brasileiros tiveram uma permanência hospitalar média de 11 dias $^{27}$. Esta diferença pode estar relacionada ao tratamento menos eficaz das doenças que levam ao aparecimento de IC e a não aderência ao tratamento preconizado que comprovadamente reduz a mortalidade e o tempo de internação desses pacientes.

Quanto à Fração de Ejeção (FE), observou-se maior prevalência de ICFEP nas mulheres em relação aos homens, que tiveram maiores taxas de ICFER. A ICFEP é mais comumente encontrada em pacientes do sexo feminino, como reportado na Diretriz Europeia de IC em $2016^{28}$, enquanto a ICFER, no sexo masculino. Observouse que a ICFEP foi mais comum em pacientes tabagistas, embora este dado não tenha significância estatística no presente estudo e com anemia, enquanto em um estudo realizado na cidade de Niteroi em 2012 esses dados foram mais prevalentes na ICFER ${ }^{29}$. Não foram encontrados dados na literatura atual que justificasse essa diferença.

Analisando os sinais e sintomas de IC de acordo com os critérios de Framingham, verificou-se que foram mais prevalentes na ICFER, achados estes que estão de acordo com os resultados encontrados por Freiberger e cols $(2015)^{30}$.

Correlacionando-se as variáveis clínico-demográficas com os dias de internação hospitalar (DIH), não se observou associação estatisticamente significativa para hiponatremia e FE reduzida, que são bem estabelecidas como fatores de mau prognóstico de acordo com a literatura ${ }^{31}$, fato este que pode ser atribuído a não avaliação da mortalidade dos pacientes na amostra estudada, uma vez que a FE reduzida e a hiponatremia podem ter contribuído com um aumento na mortalidade e consequente diminuição do tempo de internação hospitalar.

A porcentagem de pacientes que receberam IECA/BRA, betabloqueadores e espironolactona, que são medicações que foram consagradas pelas diretrizes assistenciais por possuírem grande impacto no aumento da sobrevida da ICFER, foi semelhante ao visto em outros estudos como European Society of Cardiology Heart Failure Long-Term Registry (ESC-HF$(\mathrm{LT})^{32}$, El Registro Chileno de Insuficiencia Cardiaca (ICARO) ${ }^{33}$ e BREATHE ${ }^{18}$, apesar do seu uso como terapia tríplice (IECA/BRA + betabloqueador + espironolactona) ser baixo, o que pode ser explicado pelo receio dos médicos em associar medicações como IECA/espironolactona, que aumentam os níveis séricos do potássio. Tal fato precisa ser melhorado porque alguns estudos como o Registry to Improve the Use of Evidence-Based Heart Failure Therapies in the Outpatient Setting (IMPROVE-HF) $^{34}$ mostram que a adição de cada terapia baseada em evidência está associada com importante diminuição do risco de mortalidade em 1 ano.

Dentre as limitações do presente estudo, deve-se considerar que este foi um estudo retrospectivo, dependente de revisão de prontuários e da confiabilidade dos dados nele 
existentes, com destaque a falta de um prontuário completo, não havendo informações suficientes para justificar a baixa porcentagem de pacientes fazendo uso da terapia tríplice da IC. Sendo assim, ressalta-se a importância da elaboração de medidas que visem melhorias na qualidade dos prontuários dos pacientes internados.

Poucos estudos epidemiológicos avaliaram os pacientes hospitalzados $^{15}$. Este estudo conseguiu demonstrar um panorama das internações por IC no Hospital de Referência Estadual de Alta Complexidade Dr. Carlos Macieira em São Luis-MA, além de traçar um perfil clínico-epidemiológico e descrever alguns dos principais fatores de risco e terapêutica farmacológica usada na IC.

\section{Conclusão}

De acordo com os dados apresentados, conclui-se que a IC com fração de ejeção reduzida (ICFER) foi mais associada ao sexo masculino, internação prolongada, idade avançada e sinais e sintomas dos critérios de Framingham. No grupo dos pacientes com IC com fração de ejeção preservada (ICFEP), os fatores de risco mais comuns foram tabagismo e anemia, enquanto no grupo dos pacientes com ICFER, os mais prevalentes foram DM e DRC. Quanto ao tratamento empregado na ICFER, predominou o uso de IECA (Inibidor da Enzina Conversora da Angiotensina) ou BRA (Bloqueador do Receptor da Angiotensina), espironolactona e betabloqueador.

\section{Referências}

1-Abuhab, Abrão. Análise de dados de pacientes internados por insuficiência cardíaca descompensada: impactos sobre desfechos clínicos e custos. Tese (Doutorado em Cardiologia) - Universidade de São Paulo, 2012.

2-Albuquerque. D., et. al. I Registro Brasileiro de Insuficiência Cardíaca: Aspectos Clínicos, Qualidade Assistencial e Desfechos Hospitalares. Arquivos Brasileiros de Cardiologia. Rio de Janeiro. v.104, n.6, jun.2015. Disponível em: <http://www.arquivosonline.com.br/2015/10 406/pdf/Interativa_portugues.pdf> Acesso em: $12 . f e v .2017$

3-Cowie. Martin R, et. Al.The epidemiology of heart failure. European Heart Journal v. 18, p. 208-225, 1997.

4-DATASUS. Ministério da Saúde. DATASUS. Informações de Saúde. 2008-2011. Disponível em: <http://tabnet.datasus.gov.br/cgi/ibge/popd escr.htm> Acesso em: 12.fev.2017.

5-Hsich, Eileen M, PIÑA, lleana L. Heart failure in women-A need for Prospective Data. J Am Coll Cardiol, v-4, p. 491-498, 2009.

6-Hunt, S., et al. Focused uptade incorporated into the ACC/AHA 2005 Guidelines for the Diagnosis and Management of Heart Failure in Adults A. Repor of the American College of Cardiology Foundation/American Heart Association Task Force on Practice Guidelines Developed in Collaboration With the International Society for Heart and Lung Transplantation. J Am Coll Cardiol. V. 14, n. 53, p. 1-90. Abr, 2009.

7-Lund Lars $\mathrm{H}$; Mancini, Donna. Heart failure in women. Med Clin N Am, v. 88 p. 1321-1345, 2004.

8- Mc Murray, John J. V.; STEWART S. The burden of heart failure. European Heart Journal, p.50-58, 2002.

9-Mozaffarian, D., et. al. Prediction of Mode of Death in Heart Failure. The Seattle Heart Failure Model. Circulation. v.116, p.392-398, 2007.

10-Pereira-Barreto, A.C., Suporte Avançado de vida em Insuficiência Cardíaca. São Paulo. Manole, 2015. $120 \mathrm{p}$.

11-Pina IL et. al. Exercise and heart failure: a statement from de American Heart Association Committee on Exercise, 
Rehabilitation and Prevention Circulation. v.107, p.1210-1225, 2003.

12-Yeh, Edward. T.H. et. Al. Cardiotoxicity due to Cancer Therapy. Tex Heart Inst Journal. v. 38, n.3, p.253-256, 2011.

13- Bocchi, E. et al. Sociedade Brasileira de Cardiologia. III Brazilian Guidelines on Chronic Heart Failure. Arq Bras Cardiol. 2009; 93 (1 Suppl I): 3-70

14- The Solvd Investigators. Effect of enalapril on survival in patients with reduced left ventricular ejection fractions and congestive heart failure. N Engl J Med; v. 325(5): 293302, 1991

15- Ghali, JK et al. Gender diferences in advanced heart failure: insights from the BEST study. J Am Coll Cardiol, v. 42, n. 12, p. 2128-34, dez., 2003

16- Hsich, Eileen M, Piña, Illeana L. Heart failure in women - A need for Prospective Data. J AM Coll Cardiol, 4:491-498, 2009.

17- Irma Laonigro. Et al. Alcohol abuse and heart failure. European Journal of heart failure. 200; 11: 453-462

18- Nogueira Patrícia Resende, Rassi Corrêa Krislainy de Sousa. Perfil epidemiológico, clínico e terapêutico da insuficiência cardíaca em hospital terciário. Universidade Federal de Goiás, Goiania, GO - Brasil. Arquivos brasileiros de cardiologia. 95(3), 2010.

19- Barreto ACP, Del Carlo CH, Cardoso JN, Morgado PC, Munhoz RT, Eid MO, et al. Rehospitalizações e morte por insuficiência cardíaca - índices ainda alarmantes. Arq Bras Cardiol. 2008; 91(5): 335-4

20- Gheorghiade, Mihai et al. Rehospitaliztion of Heart Failure. Problems and Perspectives. J Am Coll Cardiol. V. 6, n.4, p. 391-403, jan., 2013

21- Rabelo ER, Aliti GB, Linch GFC, Sauer JM, Mello AMFS, Martins SM. et aL. Manejo não farmacológico de pacientes com insuficiência cardíaca descompensada: estudo multicêntrico - EMBRACE*. Acta Paul Enferm. 2012;25(5):660-5.

22- Balieiro HM, Osugue RK, Rangel SP, Brandão R, Balieiro TL, Bernardez S. Perfil Clínico-Demográfico e Indicadores de Qualidade da Insuficiência Cardíaca em uma Área Rural. Arq Bras Cardiol 2009; 93(6) : 687-691

23- Neto FS, Magalhães $H M$, Batlouni $M$, Piegas LS. Inibidor da ECA e
Concentrações do Peptídeo Natriurético do Tipo B, em Idosos com Insuficiência Cardíaca. Arq Bras Cardiol 2009;92(5):349356

24- Rabelo ER, Aliti GB, Linch GFC, Sauer JM, Mello AMFS, Martins SM. et aL. Manejo não farmacológico de pacientes com insuficiência cardíaca descompensada: estudo multicêntrico - EMBRACE* ${ }^{*}$. Acta Paul Enferm. 2012;25(5):660-5.

25 - Schocken DD. Benjamin EJ, Fonarow GC, Krumholz HM, Levy D, Mensah GA. et al. Prevention of Heart Failure: A Scientific Statement From the American Heart Association Councils on Epidemiology and Prevention, Clinical Cardiology, Cardiovascular Nursing, and High Blood Pressure Research; Quality of Care and Outcomes Research Interdisciplinary Working Group; and Functional Genomics and Translational Biology Interdisciplinary Working Group. Circulation 2008;117;25442565.

26- Mangini S, Silveira FS, Silva CP, Grativvol PS, Seguro LFBC, Ferreira SMA. et al. Insuficiência Cardíaca Descompensada na Unidade de Emergência de Hospital Especializado em Cardiologia. Arquivos Brasileiros de Cardiologia 2008; 90(6): 433440.

27- Tavares LR, Heraldo Victer H, Linhares JM, Barros CM, Oliveira MV, Pacheco LC. et al.Epidemiologia da Insuficiência Cardíaca Descompensada em Niterói - Projeto EPICA - Niterói. Arquivos Brasileiros de Cardiologia 2004; 82: 121-4.

28- McMurray JJ, Adamopoulos S, Anker SD, Auricchio A, Böhm M, Dickstein K, et al; ESC Committee for Practice Guidelines. ESC guidelines for the diagnosis and treatment of acute and chronic heart failure 2012: The Task Force for the Diagnosis and Treatment of Acute and Chronic Heart Failure 2012 of the European Society of Cardiology. Developed in collaboration with the Heart Failure Association (HFA) of the ESC. Eur J Heart Fail. 2012;14(8):803-69. Erratum in: Eur J Heart Fail. 2013;15(3):361-2.

29- . Lenzen MJ, Boersma E, Reimer WJ, Balk $\mathrm{AH}$, Komajda M, Swedberg K, et al. Underutilization of evidence-based drug treatment in patients with heart failure is only partially explained by dissimilarity to patients enrolled in landmark trials: a report from the Euro Heart Survey on Heart Failure. Eur Heart J. 2005;26(24):2706-13. 
30- Fonarow GC; ADHERE Scientific Advisory Committee. The Acute Decompensated Heart Failure National Registry (ADHERE): opportunities to improve care of patients hospitalized with acute decompensated heart failure. Rev Cardiovasc Med. 2003;4 Suppl. 7:S21-30.

31- Miranda $\mathrm{CH}$, Castro RBP, Pazin Filho A. Medical management of acute decompensation of chronic heart failure in the emergency room. Circulation. 2003;36:179-86.

32- Nikolaou M, Parissis J, Yilmaz MB, Seronde MF, Kivikko M, Laribi S, et al. Liver function abnormalities, clinical profile, and outcome in acute decompensated heart failure. Eur Heart J. 2013;34(10):742-9.

33- Ribeiro, Rodrigo A. ET AL,. Custo Anual do Manejo da Cardiopatia Isquêmica Crônica no Brasil. Hospital das Clínicas de Porto Alegre, Hospital Moinhos de Vento e Universidade Federal do Rio Grande do Sul - Porto Alegre, RS 2005.

34- Filho, Albanesi . O que vem ocorrendo com a insuficiência cardíaca no Brasil? Arquivos Brasileiros de Cardiologia. São Paulo, 2005. 